EDE ECONOMIAE
章AGRONEGÓCIO

ISSN impresso: 1679-1614

ISSN online: $2526-5539$

Vol. 17 | N. 3 | 2019

Felipe Orsolin Teixeira ${ }^{2}$

ORCID: $0000-0002-6686-0164$

Elen Presotto 2

ORCID: 0000-0001-9897-7159

Clailton Ataídes de

Freitas $^{3}$

ORCID: 0000-0003-0754-3211

1 Pontifícia Universidade Católica do Rio Grande do Sul, Porto Alegre, Rio Grande do Sul, Brasil.

2 Universidade Federal do Rio Grande do Sul, Porto Alegre, Rio Grande do Sul, Brasil.

3 Universidade Federal de Santa Maria, Santa Maria, Rio Grande do

Sul, Brasil.

* patricia.batistella@edu.pucrs.br

Recebido em: 28/10/2018

Aceito em: 30/07/2019

\section{AVALIAÇÃO DO PROCESSO DE CONVERGÊNCIA DA PRODUTIVIDADE AGRÍCOLA: UMA ANÁLISE ESPACIAL DOS MUNICÍPIOS GAÚCHOS}

\section{RESUMO}

O Rio Grande do Sul (RS) é caracterizado por possuir uma vasta área rural e tem a agricultura como uma das suas principais atividades produtivas, com produtos destinados tanto para o mercado interno como também para exportação. No entanto, o crescimento dessa atividade nem sempre ocorre de maneira equilibrada entre os municípios. Com isso, o objetivo do presente estudo é identificar as heterogeneidades e analisar o processo de convergência absoluta da produtividade agrícola nos municípios do estado no período de 2001 a 2015. Para tanto, utilizou-se do ferramental econométrico espacial e da análise exploratória de dados espaciais para visualizar a dependência espacial e a formação de clusters no estado. Os principais resultados indicaram a existência de autocorrelação espacial da produtividade agrícola entre os municípios e um agrupamento de municípios com características de alta produtividade na região da Serra Gaúcha. Os modelos econométricos levaram, então, a à rejeição da hipótese de convergência da produtividade agrícola entre os municípios do estado.

Palavras-chave: Produtividade Agrícola; Convergência Absoluta; Espaço.

\section{ABSTRACT}

Rio Grande do Sul (RS) is characterized by having a vast rural area and has agriculture as one of its main productive activities with products destined both for the domestic market as well as for export. However, the growth of this activity does not always occur in a balanced way among the municipalities. Thus, the objective of the present study is to identify heterogeneities and analyze the process of absolute convergence of agricultural productivity in the municipalities of the state from 2001 to 2015. For that, we used spatial econometric tools and exploratory data analysis to visualize the spatial dependence and the formation of clusters in the state. The main results indicated the existence of spatial autocorrelation of agricultural productivity among the municipalities and a grouping of municipalities with characteristics of high productivity in the Serra Gaúcha region. The econometric models then led to the rejection of the hypothesis of convergence of agricultural productivity among the municipalities of the state.

Keywords: Agricultural Productivity; Absolute Convergence; Space.

JEL Code: E13; R12; Q10. 


\section{INTRODUÇÃO}

O Brasil é reconhecido mundialmente por sua elevada capacidade na produção de alimentos. Tal reconhecimento foi alcançado, em grande medida, devido à existência de aspectos geográficos e climáticos favoráveis. Estes, aliados ao processo de inovações tecnológicas ocorridas nos últimos quarenta anos, impulsionado por investimentos públicos e privados e políticas públicas estratégicas ${ }^{1}$, contribuíram para o aumento da produtividade do setor agrícola brasileiro (CHADDAD, 2017).

A produção agrícola se faz presente em todos os estados brasileiros, embora em alguns essa atividade seja predominante. No que tange à produção nacional, o Rio Grande do Sul se destaca no cultivo de diversos produtos agropecuários. Na agricultura, as culturas do arroz, fumo, trigo e soja ganham destaque. No ano de 2017, por exemplo, a produção estadual de arroz, fumo, trigo e soja correspondeu a $70 \%, 47 \%, 27 \%$ e $16 \%$ da produção nacional, respectivamente (IBGE, 2017).

Atualmente, o Rio Grande do Sul possui 497 municípios, e a atividade agrícola se faz presente em todas as regiões do estado. Porém, ao observar a participação das principais atividades no Valor Adicionado Bruto (VAB), é possível verificar a existência de concentrações regionais de produção, como a fumicultura nas proximidades de Santa Cruz do Sul, a sojicultura em Não-Me-Toque e Tupanciretã, a pecuária leiteira em Palmeira das Missões, e a viticultura em Caxias do Sul e Bento Gonçalves (FEE, 2017).

No ano de 2015, o município de Cachoeira do Sul, localizado na região do Vale do Jacuí, obteve o maior VAB da agropecuária sul-rio-grandense, representando $1,4 \%$ do total do estado. As principais atividades agropecuárias presentes nesse município são as produções de soja e arroz. Destacam-se, ainda, os municípios de Uruguaiana (arroz e bovinos), Tupanciretã (soja), Dom Pedrito (arroz e soja), Alegrete (arroz e bovinos), São Gabriel (soja e arroz), Itaqui (arroz), Santa Vitória do Palmar (arroz), Vacaria (soja e maçã) e São Borja (arroz e soja) (FEE, 2017).

Diante de tais dados e sabendo da importância que a atividade agrícola desempenha na economia do Rio Grande do Sul, tanto na geração de emprego e renda como também na formação de divisas por intermédio das exportações, avaliar como vem ocorrendo o seu crescimento é de extrema relevância. Assim, uma maneira utilizada pela literatura com a finalidade de avaliar, ao longo de determinado período, o comportamento de fenômenos econômicos - neste caso, o crescimento da produtividade agrícola - é através dos testes de convergência.

A hipótese de convergência conhecida como convergência absoluta ou incondicional foi desenvolvida através do modelo de crescimento de Solow na década de 1950 e mostra que as rendas per capita dos países convergem para um único estado estacionário, independentemente de

${ }^{1}$ As políticas consideradas estratégicas consistiam em: elevada disponibilidade de crédito rural, garantia de preços mínimos, subsídios à importação de insumos modernos e ampliação do sistema de assistência técnica (MELO, 1980). 
suas condições iniciais (GALOR, 1996). Uma segunda hipótese de convergência, denominada de convergência condicional, busca abarcar características que não foram imputadas à primeira e pressupõe que as rendas per capita dos países que têm características estruturais idênticas, como preferências, tecnologia, taxa de crescimento da população e taxa de poupança, convergem no longo prazo, independentemente das condições iniciais (BERTUSSI; FIGUEIREDO, 2009).

Tanto o crescimento econômico como a convergência de renda são assuntos estreitamente relacionados entre si e comumente estudados no campo econômico. Desde os estudos realizados por Baumol (1986) e Abramovitz (1986), com o objetivo de testar a hipótese de convergência de renda em nível mundial, tal tema é aprimorado tanto no campo analítico quanto no metodológico e teórico.

Além dos níveis de análise, outra característica importante que foi incorporada aos estudos de convergência refere-se aos fatores espaciais. Ou seja, aspectos como a localização geográfica passam a ser considerados. Surge também o interesse em encontrar respostas sobre a influência de uma dada região no desenvolvimento das regiões vizinhas, através do efeito transbordamento (DA SILVA et al. 2011). As primeiras pesquisas sobre convergência utilizavam-se de dados regionais e, na maioria das vezes, as unidades geográficas eram tratadas como ilhas, não possuindo relação alguma com o seu entorno (QUAH, 1995).

Com base nessas questões, o objetivo do presente estudo é identificar as heterogeneidades e analisar o processo de convergência absoluta da produtividade agrícola nos municípios do Rio Grande do Sul no período de 2001 a 2015. Para isso, utilizou-se a técnica da econometria espacial.

Com a consecução desta pesquisa, busca-se contribuir para a identificação de municípios com diferentes níveis de produtividade agrícola, o que poderá possibilitar o tratamento distinto na aplicação de políticas econômicas e sociais entre as regiões. Estudos sobre a convergência absoluta da produtividade agrícola a nível municipal ainda não foram aprofundados com a utilização da análise espacial. O presente trabalho busca preencher essa lacuna na literatura empírica acerca do tema.

Além desta introdução, o estudo é composto por mais quatro seções. A segunda seção traz um breve aparato teórico sobre convergência, bem como alguns estudos empíricos que já se utilizaram de tal análise. A terceira seção é composta pelos aspectos metodológicos, e na quarta são apresentados e discutidos os resultados. Por fim, as conclusões do trabalho estão na quinta seção.

\section{REFERENCIAL TEÓRICO}

O modelo de Solow (1956) constitui um marco importante na análise da renda per capita no longo prazo. Ele se utiliza de uma função de produção neoclássica, na qual os fatores de produção apresentam retornos decrescentes, e seu principal pressuposto é de que as economias no longo prazo caminham para steady state (SS). Para que isso ocorra, é necessário 
que o crescimento seja impulsionado pela mudança tecnológica, uma variável exógena ao modelo.

É importante destacar que, nesse modelo, a tendência é de que ocorra convergência das rendas per capita do conjunto de economias para um mesmo nível (SOLOW, 1956), o que se convencionou chamar de hipótese de convergência absoluta. Tal processo ocorre quando as medidas que determinam o estado estacionário são análogas, como: taxa de poupança, índices de progresso técnico e de aumento da população. Quando esses fatores são diferentes, chega-se à convergência condicional (GALOR, 1966). A convergência condicional pressupõe que a taxa de crescimento de cada país depende da distância relativa a seu próprio estado estacionário, ou seja, cada país ou região terá seu próprio equilíbrio e não um comum, como atribuído na convergência absoluta.

Segundo Barro e Sala-i-Martin (1992), a pressuposição básica do modelo de Solow é de que uma economia mais pobre, com baixos níveis de produto per capita e de capital, com o passar do tempo, tende a alcançar as economias ricas. Tais disparidades tendem a diminuir no momento em que a economia caminha para o estado estacionário. Assim, a convergência acontece, pois, quanto menores forem os níveis iniciais de capital e de produto, mais altas serão as taxas de crescimento.

Por muitos anos, tais suposições dos modelos tradicionais de crescimento e convergência foram alvos de críticas, principalmente pela Teoria do Crescimento Endógeno (TCE), tendo como principais precursores Romer (2006) e Lucas (1988). As críticas residiam no fato de que o modelo tradicional, proposto por Solow, não dialogava com o mundo real, e a hipótese de convergência não seguia um padrão uniforme, tal como pressupunha o modelo (LOPES, 2004).

No tocante aos estudos empíricos, é valido destacar a pesquisa seminal realizada por Baumol (1986), com a qual foi possível identificar a convergência e também a divergência de rendas per capita, no período de 1870 a 1979, de um grupo composto por dezesseis países industrializados. O estudo apontou para a existência de uma convergência menos intensa entre os países socialistas.

No início da década de 1990, Barro e Sala-i-Martin, partindo dos pressupostos do modelo de Solow, incluíram na função produção a inovação tecnológica, passando também a admitir as demais formas possíveis de medir a convergência. Os autores encontraram a existência de convergência absoluta entre os estados americanos no período de 1960 a 1985. Ainda, Sala-i-Martin (1996) obtiveram os mesmos resultados para um grupo de países da OCDE no período de 1960 a 1990.

Nos últimos anos, os estudos sobre convergência, com o intuito de se tornarem mais próximos da realidade, passaram a incorporar o método da análise espacial. Conforme destacam Magalhães et al. (2000), a análise econômica está cada vez mais focada em questões relacionadas à dimensão espacial dos problemas, característica importante quando a atenção é direcionada à convergência da renda per capita regional. 
A abordagem da Nova Geografia Econômica (NGE), preconizada pelas ideias de Krugman nos anos 90, se difere dos novos modelos de crescimento econômico. Tal abordagem passa a considerar que dois fatores são fundamentais para a explicação das desigualdades entre as regiões. O primeiro está relacionado ao espaço, que traz implicações diretas na localização das atividades, e o segundo diz respeito à distância, aspecto responsável por determinar os custos de transporte e a atração de novas atividades para determinada região (OLIVEIRA; JACINTO; GROLLI, 2008).

Com essa perspectiva de que o processo de convergência poderia ser afetado por variáveis de cunho espacial, os novos estudos passam a incorporar em seu escopo analítico a econometria espacial. Assim, a análise da convergência de renda regional passa a considerar a possibilidade de existência de dependência espacial entre as regiões. Essa abordagem não é tão recente, visto que a possibilidade de dependência espacial foi considerada no tratamento da convergência regional no final da década de 1990, por Rey e Montouri (1999), que estudaram a convergência de renda dos Estados Unidos.

Na Espanha, Dall'erba (2003) buscou identificar, para 48 regiões, no período de 1980 a 1996, as disparidades da produtividade do trabalho. O estudo utilizou-se dos conceitos de $\beta$ - convergência e $\sigma$-convergência, bem como da metodologia de econometria espacial. Os resultados obtidos destacam a importância de levar em consideração o impacto da produtividade dos locais vizinhos.

Destacado como um dos principais trabalhos na área de economia brasileira sobre o tema em questão, Ferreira e Ellery Jr. (1996) buscaram analisar a existência de convergência entre a renda per capita dos estados brasileiros, nos anos de 1970 a 1985, embasados na metodologia proposta por Barro e Sala-i-Martin (1990). Os principais resultados encontrados identificaram um processo de convergência mais lento do que o encontrado por estes autores para os estados americanos.

Monastério e Ávila (2004) buscaram identificar a existência de convergência de renda em cinquenta e oito áreas estatisticamente comparáveis no Rio Grande do Sul, por meio da utilização de técnicas de econometria espacial. A análise exploratória de dados espaciais (AEDE) possibilitou identificar que municípios com elevado nível de crescimento estão localizados na Região da Serra, enquanto os menos dinâmicos estão localizados na Região da Campanha. Além disso, evidenciaram que tais áreas possuem vizinhança com características que demonstram o mesmo padrão de renda.

A investigação da hipótese de convergência da produtividade também já foi alvo de estudos no Brasil. Lopes (2004) analisou a evolução da produtividade da terra para as onze principais culturas agrícolas brasileiras e utilizou como modelo de análise a hipótese de convergência da produtividade nos estados brasileiros. Os resultados apontaram que apenas seis, das onze culturas, confirmaram a hipótese de convergência absoluta. Além disso, o estudo identificou a necessidade da implantação 
de políticas econômicas que possam contribuir para que esse processo ocorra, principalmente, nas culturas do arroz e do milho.

$\mathrm{Na}$ tentativa de testar a hipótese de convergência da produtividade do trabalho no setor agropecuário dos estados brasileiros, Fochezatto e Stülp (2008) utilizaram-se da técnica de cadeias de Markov, a fim de comparar o setor agropecuário com os demais setores, bem como avaliar seu comportamento ao longo da década de noventa. Os resultados rejeitaram a existência de convergência da produtividade do trabalho no setor agropecuário, apontando que alguns estados evoluem para um nível superior de produtividade, enquanto outros para um nível inferior.

Almeida (2008) analisou a hipótese de convergência da produtividade agrícola para as microrregiões brasileiras nos anos de 1991 a 2003, utilizando-se dos pressupostos desenvolvidos por Baumol (1986). O estudo identificou, por meio do método de econometria espacial, a existência de convergência absoluta da produtividade agrícola brasileira, sendo que esta converge de maneira muito lenta para o steady state.

Frente a esses estudos empíricos já realizados, o trabalho que se desenha busca trazer contribuições para o debate sobre convergência da produtividade agrícola no Rio Grande do Sul. Sua pertinência reside, principalmente, na análise, até então não realizada, dessa variável, considerando os municípios gaúchos, e também na utilização da econometria espacial, a qual tem por finalidade incluir características espaciais.

\section{METODOLOGIA}

A seção a seguir traz os aspectos metodológicos empregados, bem como o tratamento dos dados utilizados para a análise da convergência da produtividade agrícola dos municípios do Rio Grande do Sul.

\section{Base de dados}

Os dados utilizados para a análise da convergência da produtividade agrícola dos municípios do Rio Grande do Sul foram extraídos junto à Produção Agrícola Municipal (PAM), do banco de dados do Sistema de Recuperação Automática (SIDRA) do IBGE, referentes aos anos de 2001 a 2015. Esse recorte de tempo foi determinado por abranger o maior número de municípios². O Rio Grande do Sul possui 497 municípios (IBGE, 2017) e na análise são considerados apenas aqueles que possuem rendimento agrícola, totalizando $477^{3}$.

\footnotetext{
2 Até o ano de 1999, o Rio Grande do Sul teve muitos municípios emancipados, sem dados para o período anterior, cuja produção foi somada à dos municípios que ainda não haviam sido desmembrados, fato que, se considerado, geraria um certo viés na análise.

3 Os municípios que não possuem produtividade agrícola são: Arroio do Sal, Barra do Quaraí, Pedro Osório, Santa Margarida do Sul, Tramandaí, Pinto Bandeira, Alvorada, 
Segundo Almeida (2008), as variáveis absolutas podem levar a erros na intepretação dos resultados, pois há correlação com o tamanho do município em estudo. A variável produtividade média agrícola foi relativizada pela razão entre o valor da produção ${ }^{4}$ das lavouras temporária e permanente e a área plantada e destinada à colheita, respectivamente. A explicação para a utilização da área plantada, no caso de lavoura temporária, e área destinada à colheita, para o caso da lavoura permanente, deve-se ao fato de que, na agricultura, a decisão do uso de insumos é definida antes que o produto seja cultivado, o que leva a um planejamento de produção por parte dos agricultores. Assim, as variáveis área plantada e área destinada à colheita consistem em proxies para uma ponderação adequada de produtividade média (ALMEIDA, 2005).

O valor da produção foi deflacionado através do Índice de Preços por Atacado $^{5}$ (IPA), calculado pela Fundação Getúlio Vargas (FGV). O ano base utilizado foi o de 2015 .

\section{Análise exploratória de dados espaciais}

A econometria espacial busca em si, trazer à tona, a influência dos fatores geográficos e de localização espacial no crescimento e desenvolvimento das regiões. Dessa maneira, o incremento das ferramentas de análise exploratória de dados espaciais constitui um passo importante a ser realizado a priori, ou seja, antes de proceder a estimação do modelo econométrico, considerando que tal procedimento irá contribuir para que se tenha uma análise estatística sofisticada. A AEDE, bem como as técnicas econométricas espaciais, torna possível a obtenção de uma nova percepção da dinâmica geográfica dos padrões de crescimento da renda no tempo.

A análise exploratória de dados espaciais (AEDE) constitui um conjunto de técnicas que tem por finalidade identificar e descrever distribuições espaciais. Visa detectar a existência ou não de localizações espaciais atípicas, outliers, padrões de associação espacial, formação de clusters ou demais formas de heterogeneidade espacial (BAUMONT; ERTUR; LE GALLO, 2000). Em suma, a AEDE tem por objetivo identificar se há ou não dependência espacial. Além disso, ela irá contribuir para que se consiga uma especificação mais adequada da forma funcional.

O primeiro passo a ser realizado, num estudo em que a AEDE é utilizada, é testar se os dados são distribuídos aleatoriamente ou apresentam dependência espacial. A aleatoriedade espacial supõe que os valores de uma característica de determinada região não dependem dos valores das mesmas características das regiões contíguas. Tal hipótese poderá ser

Balneário Pinhal, Canoas, Capivari do Sul, Cidreira, Esteio, Palmares do Sul, Sapucaia do Sul, Tavares, Xangri-lá, Imbé, Chuí, Cambará do Sul e Cachoeirinha.

4 Produção obtida multiplicada pelo preço médio ponderado (IBGE, 2017).

5 O IPA tem por base as pesquisas estruturais relativas aos setores agropecuário e industrial, além das Contas Nacionais, divulgadas pelo IBGE. Para compor a parcela agropecuária do IPA, são selecionados produtos pertencentes a três classes de atividades: lavouras temporárias, lavouras permanentes e pecuária. 
identificada através das estatísticas de autocorrelação espacial (ALMEIDA, 2012).

Conforme Almeida (2012), para implementação da AEDE, é necessário que, primeiramente, seja definida uma matriz de pesos espaciais $(\boldsymbol{W})$, responsável por apresentar os arranjos espaciais das interações resultantes do fenômeno que está sendo estudado. Em suma, a matriz $(\boldsymbol{W})$ indica o quanto a interação é mais forte no caso das regiões mais próximas geográfica ou economicamente, e mais fraca nas regiões mais distantes. Essa matriz tem por finalidade capturar toda autocorrelação espacial presente no fenômeno estudado. Trata-se de uma matriz quadrada e os pesos espaciais $\boldsymbol{W}_{\boldsymbol{i} \boldsymbol{j}}$ representam a influência do município $j$ sobre o município $i$. Assim, por convenção, $\boldsymbol{W}_{\boldsymbol{i} i}$ é igual a zero.

A matriz utilizada poderá assumir diferentes formas: por contiguidade, a qual representa a ideia de fronteira física e se divide em três tipos - rainha, bispo e torre; por distância geográfica; ou por uma variável socioeconômica. O critério de definição para escolha é baseado no maior I de Moran significativo.

A autocorrelação espacial global pode ser definida como a coincidência entre similaridade de valor e similaridade locacional. Dessa forma, não há autocorrelação espacial positiva quando altos ou baixos valores de uma variável aleatória se aglomeram no espaço, e não há autocorrelação espacial negativa quando áreas geográficas tendem a ser cercadas por vizinhos com valores muito diferentes (BAUMONT; ERTUR; LE GALLO, 2000).

A estatística de autocorrelação espacial é resultante da composição de três elementos: uma medida de autocovariância, uma medida de variância dos dados e a matriz de ponderação espacial $(\boldsymbol{W})$. As principais medidas adotadas na literatura para obtenção dos indicadores de autocovariância são: a estatística $I$ de Moran, a estatística $c$ de Geary e a estatística $G$ de Getis-Ord (ALMEIDA, 2012).

A estatística I de Moran foi desenvolvida por Moran em 1948 e é comumente a mais utilizada. Moran (1948) construiu um coeficiente de autocorrelação espacial utilizando a medida de autocovariância na forma de produto cruzado. Matricialmente, a estatística é dada por:

$$
I=\frac{n}{S_{o}} \frac{Z^{\prime} W_{Z}}{Z^{\prime} Z}
$$

em que $n$ é o número de regiões; $Z$ denota os valores da variável de interesse padronizada; $W_{Z}$ representa os valores médios da variável de interesse padronizada nos municípios vizinhos, os quais são definidos por uma matriz de ponderação espacial $W ; S_{o}$ representa a operação $\sum_{i} \sum_{j} W_{i j}$, indicando que todos os elementos da matriz de pesos espaciais $W$ devem ser somados; e o termo do numerador $Z^{\prime} W_{Z}$ é a autocovariância espacial, composta pelos seus produtos cruzados. A estatística I de Moran, de acordo com Almeida (2012), é uma espécie de coeficiente de 
autocorrelação, representando a relação da autocovariância do tipo produto cruzado com a variância dos dados $\left(Z^{\prime} Z\right)$.

Os valores do $I$ de Moran superiores a $-1[1 /(n-1)]$ indicam que há autocorrelação espacial positiva, ou seja, altos valores, em uma determinada região, são incorporados por vizinhos com características semelhantes. $O$ índice menor que o valor crítico sugere que existe autocorrelação espacial negativa, ou seja, altos valores estão localizados perto de valores baixos, ou vice-versa (ALMEIDA, 2012).

O diagrama de dispersão de Moran é dividido em quatro quadrantes, sendo eles: Baixo-Baixo, Alto-Baixo, Baixo-Alto e Alto-Alto. Com ele, é possível identificar o padrão de associação local espacial existente entre os municípios e seus vizinhos (ALMEIDA, 2012). Além disso, a reta de inclinação pode ser traçada, o que torna mais fácil a identificação da existência de possíveis outliers e do modo como influenciam o valor do I de Moran.

Além das estatísticas globais já apresentadas, também é interessante avaliar os indicadores de autocorrelação espacial locais. Apesar de existir mais de uma opção para detectar os padrões de associações locais, o trabalho utiliza-se da estatística I de Moran local.

Anselin (1995) propõe um indicador capaz de capturar padrões locais de autocorrelação espacial estatisticamente significativos, denominando-o de Local Indicator of Spatial Association (LISA). Tal estatística calcula um $I_{i}$ para cada observação. Assim, tem-se $n$ computações da estatística $I_{i}$ juntamente com seus níveis de significância. Entretanto, como são obtidas inúmeras informações, uma para cada observação, a melhor maneira encontrada para apresentá-las é mapeando o conjunto de estatísticas que são obtidas através do mapa de significância LISA.

O indicador I de Moran local pode ser visualizado de três maneiras: através do diagrama de dispersão de Moran e também por meio de mapas de significância LISA e de clusters LISA. O mapa de significância LISA apresenta apenas as observações que foram significativas de acordo com o seu valor de probabilidade. Já o mapa de clusters LISA, combina as informações do diagrama de dispersão de Moran e do mapa de significância das medidas de associação local.

\section{Modelo Econométrico Espacial}

Os modelos econométricos lineares não levam em conta o espaço em seus parâmetros. Com isso, quando um modelo possui, em seu contexto teórico e empírico, a influência do espaço, o melhor método a ser utilizado é aquele por meio do qual se consegue capturar tal efeito. Para isso, no modelo de regressão linear clássico (Equação 2), são inseridos componentes espaciais.

$$
\mathbf{y}=\mathbf{X} \boldsymbol{\beta}+\boldsymbol{\varepsilon}
$$

$\mathrm{Na}$ equação acima, y é o vetor $n$ por 1 das observações da variável dependente, $X$ é a matriz $n$ por $k$ das variáveis explicativas exógenas, $\beta$ é o 
coeficiente de regressão e $\varepsilon$ é o vetor $n$ por 1 de termos de erros aleatórios idêntica e independentemente distribuídos (i.i.d.).

No entanto, se observada a dependência espacial, o modelo de Mínimos Quadrados Ordinários (MQO) passa a ser enviesado, sendo ideal a utilização de um modelo que leva em conta os efeitos dessa autocorrelação espacial. Entre os principais modelos, a dependência espacial pode estar no termo de erro (SEM), na variável dependente (SAR) ou no erro e na variável dependente (SARMA). No entanto, o SARMA analisa o termo de erro local com influência apenas sobre os seus vizinhos mais próximos. As Equações 3 a 5 representam os modelos SAR, SEM e SARMA, respectivamente.

$$
\begin{gathered}
y=\rho W y+\varepsilon \\
y=X \beta+\lambda W \mu+\varepsilon \\
y=\rho W 1 y+X \beta+\theta W 2 \varepsilon+\varepsilon
\end{gathered}
$$

Tem-se que $\rho$ é o coeficiente de defasagem autorregressivo espacial; $\mathbf{W y}$ é o vetor de defasagens espaciais na variável dependente $(n \times 1) ; \lambda$ é o parâmetro do erro autorregressivo espacial, que acompanha a defasagem $\mathbf{W} \boldsymbol{\mu}$ (dependência espacial no termo de erro); e $\boldsymbol{\theta}$ é o vetor do coeficiente autorregressivo para os erros locais da regressão. Cabe destacar que W1 e W2 podem ser matrizes com pesos espaciais diferentes.

Para estimar a convergência da produtividade agrícola, utiliza-se a Equação 6, conforme proposto por Almeida et al. (2008).

$$
\ln \left(R m e_{t} / R m e_{t-n}\right)=\propto+\beta \ln \left(R m e_{t-n}\right)+u_{i}
$$

em que $\ln \left(R m e_{t} / R m e_{t-n}\right)$ é o logaritmo natural da divisão da renda média agrícola entre os dois anos em análise, $\ln \left(R m e_{t-n}\right)$ é o logaritmo natural da renda média agrícola no período inicial do estudo e $u_{i}$ é o termo de erro.

Primeiramente, o modelo é estimado via método dos Mínimos Quadrados Ordinários (MQO), a fim de identificar qual a melhor maneira de calcular a Equação 6. Alguns modelos econométricos espaciais são: de Defasagem Espacial, Erro Autoregressivo Espacial, Erro de Média Móvel Espacial, Componente de Erro Espacial, Defasagem Espacial com Erro Autoregressivo Espacial e Regressivo Cruzado Espacial (ALMEIDA, 2012).

Florax, Folmer e Rey (2003) especificam, de forma resumida, tais procedimentos, indicando os seguintes passos: i) estimar o modelo por MQO; ii) testar a hipótese de não dependência espacial por meio do

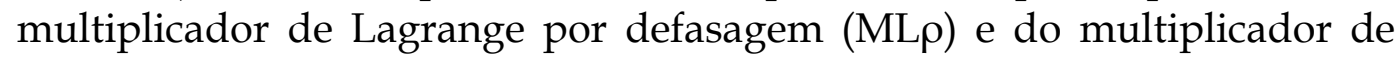

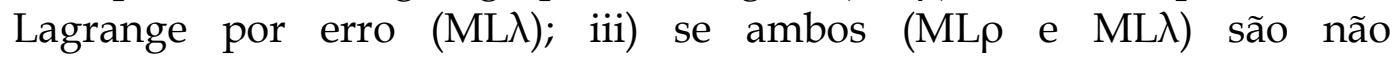
significativos, MQO será o modelo melhor especificado; iv) se ambos (ML $\rho$ e ML $\lambda$ ) são significativos, deverá ser estimado o que apresentou maior significância estatística. 


\section{Procedimentos}

A fim de verificar a autocorrelação espacial dos resíduos da regressão, é utilizado o teste I de Moran. A estatística contempla a soma dos produtos cruzados dos resíduos para as regiões vizinhas (ALMEIDA, 2012). A hipótese nula a ser testada é a de que os resíduos da regressão estimada por MQO são distribuídos aleatoriamente ao longo do espaço. Para que os resíduos estejam autocorrelacionados e a técnica econométrica espacial se faça necessária, a hipótese nula deve ser rejeitada. Segundo Anselin (2005), três testes básicos devem ser utilizados para analisar a consistência dos parâmetros gerados, sendo eles: de multicolinearidade, de normalidade dos resíduos (Jaque-Bera) e de heterocedasticidade (Breusch-Pagan, Koenker-Bassett e White).

O teste de multicolinearidade não é estático, e valores inferiores a 30 podem ser considerados apropriados ao modelo (ALMEIDA, 2012). O teste Jaque-Bera para verificar a normalidade dos resíduos possui a seguinte definição:

$H_{0:}$ A distribuição é normal;

Caso a hipótese nula do teste seja rejeitada, há a evidência de a distribuição dos resíduos não seguir distribuição normal. Para os testes de heterocedasticidade, a hipótese a ser testada é:

$H_{0:}$ Variância constante, homocedasticidade;

Se a hipótese nula for rejeitada, identifica-se que há heterocedasticidade no modelo. Nos casos em que os resíduos não são normais e o modelo apresenta heterocedasticidade, existem alguns métodos robustos a tais problemas, como o uso de variáveis instrumentais e o Método dos Momentos Generalizados (MMG).

\section{RESULTADOS E DISCUSSÃO}

\section{Análise da dependência e clusters espaciais}

O primeiro passo a ser dado na AEDE é verificar a existência de autocorrelação espacial através da análise do I de Moran. Conforme já mencionado na seção metodológica, esse índice mostra a associação espacial global e local e, caso apresente valores positivos, indica a existência de autocorrelação espacial positiva. Dessa forma, a autocorrelação espacial global univariada foi calculada para a variável produtividade agrícola nos anos de 2001, 2008 e 2015, e pode ser observada na Figura 1. 


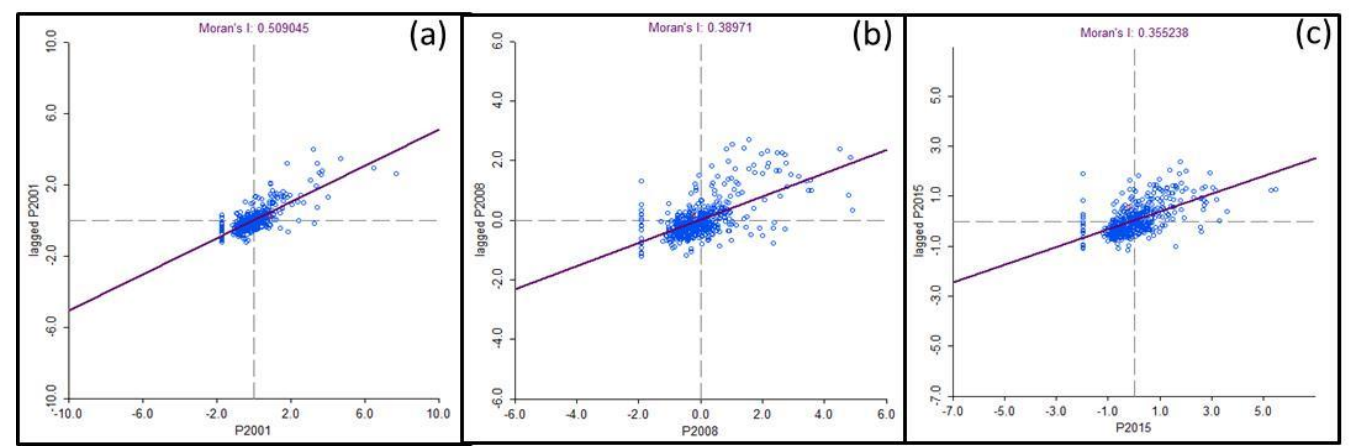

Figura 1. Estatística $I$ de Moran para a produtividade agrícola nos anos de 2001 (a), 2008 (b) e 2015 (c)

Fonte: Dados da pesquisa.

A estatística $I$ de Moran corresponde ao valor de $-0,0021^{6}$, sendo que, nos três anos analisados, 2001, 2008 e 2015, o índice calculado foi maior que o esperado: 0,50, 0,38 e 0,35, respectivamente. Existe uma similaridade entre a produtividade agrícola nos municípios gaúchos, visto que os que apresentam altos valores de produtividade estão próximos uns dos outros, o que também pode acontecer com aqueles que manifestam valores baixos. Segundo Almeida (2012), esse padrão ocorre quando há um efeito transbordamento do fenômeno em estudo, ou seja, quando o valor da produtividade por hectare de um município exerce influência em seus vizinhos. No entanto, é possível visualizar uma tendência de queda desses efeitos entre 2001 e 2015.

Os padrões globais dos testes muitas vezes não retratam os níveis locais, especialmente quando se trata de um conjunto maior de observações, como é o caso em estudo. Podem haver clusters que constituem um subconjunto pequeno de municípios em relação ao conjunto total do Rio Grande do Sul. Dessa forma, na Figura 2, é apresentado o mapa de agrupamentos LISA, que combina as informações do diagrama de dispersão de Moran e a significância dos grupos locais de $I_{i}$. 


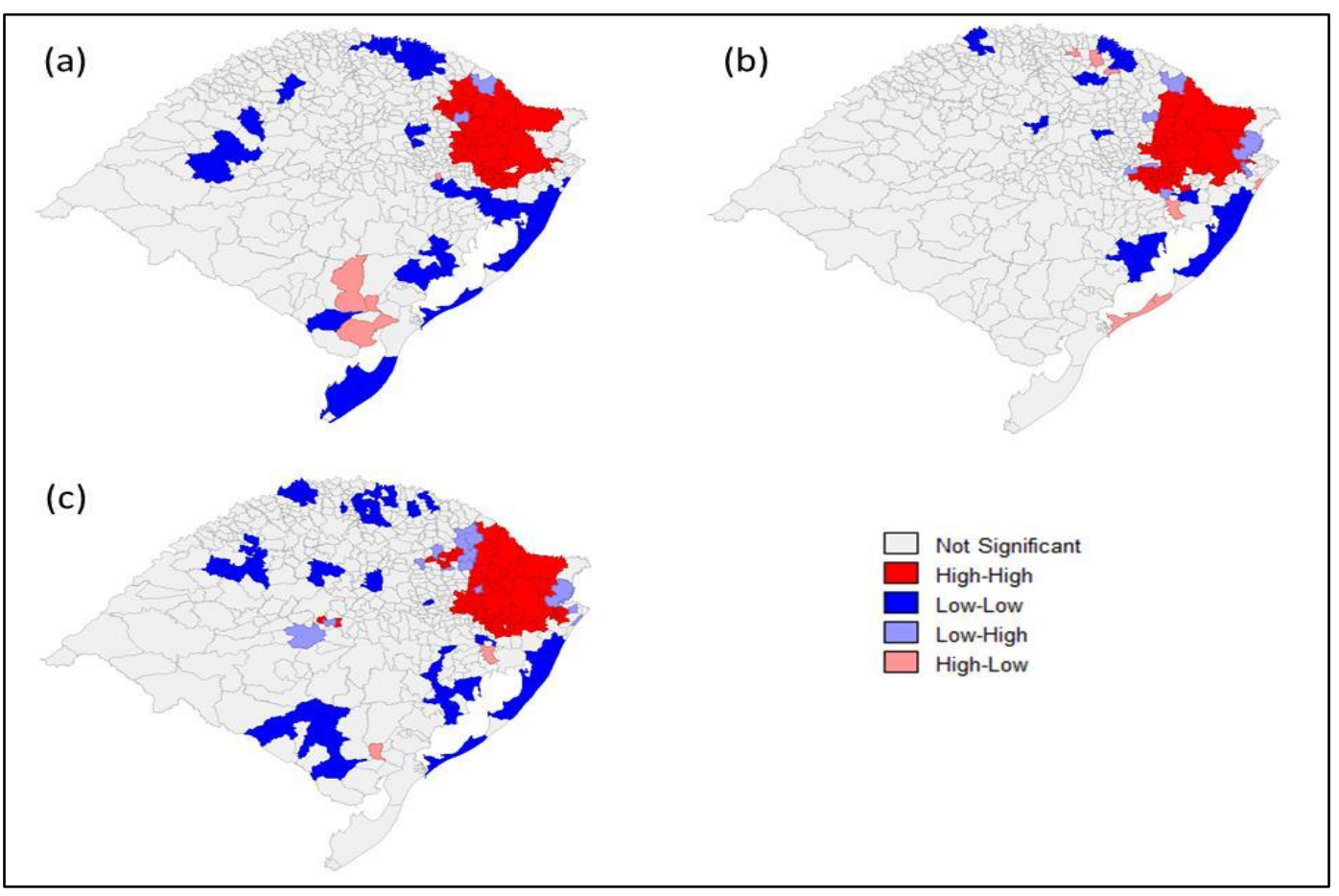

Figura 2. Mapa de clusters LISA dos municípios gaúchos nos anos de 2001 (a), 2008 (b) e 2015 (c)

Fonte: Dados da pesquisa.

Ao observar a Figura 2, verifica-se que o mapa (a), ao nível de significância estatística de $5 \%$, mostra a formação de um cluster no quadrante Alto-Alto (AA), nove clusters no Baixo-Baixo (BB), e dois clusters tanto no quadrante Alto-Baixo (AB) quanto no quadrante Baixo-Alto (BA). Os demais municípios - trezentos e oitenta e cinco - não se enquadraram nesses padrões devido ao fato de os valores para o I de Moran não serem significativos.

A lavoura temporária ocupa mais de nove milhões de hectares no estado do Rio Grande do Sul (FEE, 2017), sendo que a maior parte dessa proporção de terras volta-se especialmente para produção de grãos, tanto cereais quanto oleaginosas. As principais culturas, em termos de quantidade produzida e área plantada nas lavouras gaúchas, são de arroz, milho e trigo, as quais se espraiam em todas as regiões do estado.

O cluster AA, evidenciado pela cor vermelha, compreende 51 municípios localizados na Região Nordeste do estado, abrangendo as microrregiões de Vacaria, Montenegro, Passo Fundo, Gramado-Canela, Porto Alegre e Caxias do Sul. As principais atividades agrícolas presentes em tais regiões são as produções de milho e soja, horticultura e vitivinicultura, principalmente nas microrregiões da Serra, Gramado-Canela e Caxias do Sul.

Já no quadrante BB do mapa (a), é possível identificar a formação de seis clusters. O primeiro está localizado na Região Noroeste do estado, principalmente nas microrregiões de Erechim e Frederico Westphalen, as quais, no ano de 2001, obtiveram destaques na produção de feijão e milho. 
A soma das duas microrregiões corresponde a 33\% da produção de feijão e $17 \%$ da produção de milho do estado.

Os municípios que possuem autocorrelação negativa são Esmeralda e André da Rocha, destacados no mapa (a) pela cor azul clara, os quais apresentam um baixo valor de produtividade agrícola, embora seus vizinhos se diferenciem por possuírem uma elevada produção.

No mapa (b) da Figura 2, o cluster AA se expande para outros municípios das microrregiões já verificadas no ano de 2001, abrangendo 49 localidades. O que se nota, no ano de 2008, é um aumento das cidades com baixa renda agrícola ao redor desse agrupamento de alta produtividade. $\mathrm{O}$ número de municípios com baixa produtividade, rodeados por vizinhos com as mesmas características, diminuiu 30\%, se comparado ao mapa (a). Os municípios da Região Sudeste e Sudoeste do Rio Grande do Sul não apresentaram significância estatística para tal cluster.

Os municípios de São José do Norte, localizado no Sudeste do estado, e Capão da Canoa, na Região Metropolitana de Porto Alegre, passaram de baixa produtividade agrícola para alta, com vizinhos próximos registrando uma baixa produção. Isso se deve, fundamentalmente, ao aumento de $300 \%$ na variação da produtividade agrícola desses municípios, entre os anos de 2001 e 2008. Surgiram, então, dois novos clusters BB: o primeiro localizado em Quinze de Novembro e Selbach, e o outro formado por cinco municípios da Região Noroeste.

O mapa (c) da Figura 2 apresenta dois clusters para o quadrante AA. A localização do primeiro agrupamento se concentra na mesma região dos anos anteriores, abrangendo, em 2015, um número maior de municípios que apresentaram valor $I$ de Moran significativo individualmente. $O$ segundo agrupamento é composto por Ivorá e Dona Francisca, localizados na Região Centro Ocidental Rio-Grandense.

O número de municípios pertencentes ao quadrante BB também aumentou na análise de 2015. Um novo agrupamento surgiu abrangendo os municípios de Erval, Caçapava do Sul, Pinheiro Machado e Bagé, localizados no Sudoeste do estado. Vizinho a esses municípios, Cerrito possui um alto valor de renda agrícola e compõe o quadrante AB (AltoBaixo), juntamente com Porto Alegre.

Próximo ao novo cluster AA, na Região Central do estado, estão localizados os municípios de Faxinal do Soturno e Santa Maria, com valores significativos para o I de Moran. Esses dois municípios possuem baixa produtividade agrícola, embora seus vizinhos apresentem característica oposta.

A existência de um cluster AA na Região da Serra gaúcha, entre os anos de 2001 e 2015, já foi identificada por Monastério e Ávila (2004) em seu estudo sobre a renda dos municípios gaúchos para os anos de 1939 a 2001. Contudo, como já mencionado anteriormente, não foram encontrados trabalhos para a análise exploratória de dados espaciais a nível municipal. 
No entanto, percebe-se que as principais formações de clusters com padrões BB estiveram concentradas nas regiões mais extremas do estado, tanto no Norte quanto no Sul. A produção de soja é predominante na Região Norte, principalmente nas microrregiões Nordeste, Alto Jacuí, Rio da Várzea e Produção. Nos últimos anos, sem menosprezar os demais estados brasileiros, foi no Rio Grande do Sul que a produção da oleaginosa mais avançou e, ao observar o ano de 2006, verifica-se que essa cultura foi responsável por mais de $40 \%$ do crescimento do Valor Bruto da Produção agropecuária gaúcha. Em grande medida, esse aumento foi incentivado, principalmente, pelo crescimento da demanda externa e pela alta nos preços recebidos pelos agricultores.

Em contrapartida, na Região Sul do estado, tem-se a predominância do cultivo de arroz. Tal cultura é encontrada, principalmente, nas microrregiões Campanha e Fronteira Oeste. A produção de fumo também é destaque no Rio Grande do Sul, e os municípios com os maiores volumes de produção estão localizados nas microrregiões Centro Sul e Vale do Rio Pardo.

Percebe-se que as formações de clusters caracterizados pelos padrões AA envolveram municípios com maior proporção de lavouras permanentes, principalmente dedicadas à produção de uva, como no caso da microrregião de Caxias do Sul. Enquanto isso, de modo contrário, as regiões que são marcadas por terem maior proporção de lavouras temporárias enquadraram-se no padrão BB. Em grande medida, a produção dessas regiões é mais afetada do que a das lavouras permanentes, já que as intempéries climáticas afetam principalmente as lavouras de verão, como no caso do arroz, fumo, milho e soja, contribuindo para o aumento da volatilidade da produção de grãos.

\section{Análise do Modelo econométrico para Convergência absoluta}

Para que a hipótese de convergência seja aceita, é necessário que o parâmetro $\beta$ apresente sinal negativo. Tal resultado indica que os municípios que inicialmente detinham níveis de produtividade menores, no decorrer do período analisado, obtiveram maiores taxas de crescimento. Seguindo os procedimentos apresentados na seção metodológica, primeiramente é realizada a estimação via método dos Mínimos Quadrados Ordinários (MQO), demonstrada na Tabela 1 a seguir. 
Tabela 1. Resultados dos modelos espaciais para os subperíodos 20012007 e 2008-2015, e para o período de 2001-2015

\begin{tabular}{cccc}
\hline Coeficientes & $2001-2007^{7}$ & $2008-2015^{8}$ & $2001-2015^{9}$ \\
\hline Constante & $-0,173993$ & 0,169799 & 0,318586 \\
& $(0,03014)^{*}$ & $(0,02935)$ & $(0,00061)$ \\
\hline$\beta$ & 0,213423 & 0,021935 & 0,107461 \\
& $(0,0000)$ & $(0,01066)$ & $(0,0000)$ \\
\hline AIC & 395,239 & 357,815 & 537,783 \\
\hline SC & 403,652 & 366,228 & 546,196 \\
\hline I de Moran & 16,7592 & 13,4769 & 9,6922 \\
& $(0,0000)$ & $(0,0000)$ & $(0,0000)$ \\
\hline ML - Erro & 272,8007 & 169,6301 & 90,4709 \\
& $(0,0000)$ & $(0,0000)$ & $(0,0000)$ \\
\hline ML - Defasagem & 153.6161 & 166,5760 & 65,8578 \\
& $(0,0000)$ & $(0,0000)$ & $(0,0000)$ \\
\hline MLR - Erro & 119,3116 & 3,1465 & 28,3533 \\
& $(0,0000)$ & $(0,07609)$ & $(0,0000)$ \\
\hline MLR - Defasagem & 0,1269 & 0,0924 & 3,7401 \\
& $(0,72163)$ & $(0,76114)$ & $(0,05312)$ \\
\hline Teste Breusch-Pagan & 13,9801 & 19,1362 & 5,9711 \\
& $(0,00018)$ & $(0,00001)$ & 0,01454 \\
\hline Teste Jarque-Bera & 38,1082 & 78,5697 & 17,6835 \\
& $(0,0000)$ & $(0,0000)$ & $(0,00014)$ \\
\hline
\end{tabular}

Fonte: Dados da pesquisa.

Nota: ${ }^{*}$ P-valor; $\mathrm{ML}=$ Multiplicador de Lagrange; MLR $=$ Multiplicador de Lagrange Robusto.

Nos modelos estimados por MQO, foi possível verificar a existência de autocorrelação espacial dos erros da regressão (o parâmetro I de Moran é estatisticamente significativo), ou seja, a produção agrícola dos municípios gaúchos possui dependência espacial. Com isso, volta-se a análise para os valores dos Multiplicadores de Lagrange (LM), a fim de verificar qual é a melhor especificação espacial a ser realizada, visto que, para os três períodos analisados, os testes $L M$ e $L M$ Robusto do Erro Espacial foram significativos, indicando que o modelo mais apropriado é o do Erro Espacial (spatial error models - SEM).

Os testes estatísticos indicados por Anselin (2008) apontaram problemas de não normalidade dos resíduos (Teste de Jarque-Bera) e de heterocedasticidade (Teste Breusch-Pagan). Segundo Almeida (2008), para minimizar tais problemas, deve-se proceder com a estimação do modelo SEM, conforme apresentado na Tabela 2.

\footnotetext{
${ }^{7}$ Matriz de ponderações pelo método de contiguidade rainha.

${ }^{8}$ Matriz de ponderações pelo método de vizinhos mais próximos.

${ }_{9}^{9}$ Matriz de ponderações pelo método de contiguidade torre.
} 
Tabela 2. Resultados dos modelos espaciais (SEM) para os subperíodos 2001-2007 e 2008-2015, e para o período de 2001-2015

\begin{tabular}{cccc}
\hline Coeficiente & $2001-2007^{10}$ & $2008-2015^{11}$ & $2001-2015^{12}$ \\
\hline Constante & $-0,159605$ & 0,217128 & 0,180031 \\
& $(0,04735)$ & $(0,00709)$ & $(0,06871)$ \\
\hline$\alpha$ & 0,683373 & 0,510571 & 0,494168 \\
& $(0,0000)$ & $(0,0000)$ & $(0,0000)$ \\
\hline$\beta$ & 0,209999 & 0,0162085 & 0,123627 \\
& $(0,0000)$ & $(0,05826)$ & $(0,0000)$ \\
\hline AIC & 198,895 & 271,408 & 465,361 \\
\hline SC & 207,308 & 279,821 & 473,775 \\
\hline Teste Breusch-Pagan & 6,2649 & 9,3574 & 8,1688 \\
& $(0,01232)$ & $(0,00222)$ & 0,00426 \\
\hline
\end{tabular}

Fonte: Dados da pesquisa.

Verificado o problema de ausência de normalidade nos erros, a estimação do modelo SEM foi realizada através do Método de Momentos Generalizados (MMG), proposto por Kelejiane Prucha (1990). Tal método prescinde da hipótese de normalidade dos erros, dando maior robustez ao modelo estimado. Os resultados são apresentados na Tabela 3 a seguir.

Tabela 3. Resultados econométricos do Método de Momentos Generalizados (MMG) para os subperíodos 2001-2007 e 2008-2015, e para o período de 2001-2015

\begin{tabular}{cccc}
\hline Coeficiente & $2001-2007$ & $2008-2015$ & $2001-2015$ \\
\hline Constante & -0.1580868 & 0.2152562 & 0.1710861 \\
& $(0.0197171)$ & $(0.0006119)$ & $(0.0212802)$ \\
\hline$\alpha$ & 0.6546828 & 0.5191370 & 0.4896756 \\
& $(0.0000000)$ & $(0.0000000)$ & $(0.0000000)$ \\
\hline$\beta$ & 0.2100601 & 0.0164511 & 0.1248136 \\
& $(0.0000000)$ & $(0.0189527)$ & $(0.0000000)$ \\
\hline
\end{tabular}

Fonte: Dados da pesquisa.

Como é possível verificar, não ocorreram alterações significativas nos valores dos parâmetros, o que pode ser explicado pelo fato de o trabalho possuir uma grande amostra. A estimativa do parâmetro $\beta$, apresentado nas Tabelas 2 e 3, para todos os períodos analisados, indicou a existência de um padrão de divergência de produtividade agrícola dos municípios gaúchos.

O parâmetro positivo da produtividade inicial indica a presença de divergência da taxa de crescimento da produtividade nos municípios gaúchos, já que a expectativa proposta pela literatura é de que o

\footnotetext{
${ }^{10}$ Matriz de ponderações pelo método de contiguidade rainha.

${ }^{11}$ Matriz de ponderações pelo método de contiguidade rainha.

12 Matriz de ponderações pelo método de contiguidade torre.
} 
coeficiente assuma valor negativo. Tal resultado mostra que, no decorrer dos anos, houve um aumento da desigualdade da produção agrícola entre os municípios gaúchos, indicando que aqueles que possuem inicialmente um nível de produção maior estão crescendo em maior proporção do que os que apresentam menor produtividade.

Todos os parâmetros mostraram-se significativos ao nível de confiança de $1 \%$. O coeficiente $\alpha$ é o parâmetro do erro autoregressivo espacial e se mostrou significativo. Tal parâmetro é responsável por indicar que a variável dependente $(y)$ é influenciada pelos choques vindos de todas as regiões, isto é, do sistema como um todo, sendo que as regiões mais próximas são as mais impactadas, e o choque vai ocorrendo de maneira decrescente.

No caso da produtividade do setor agrícola, municípios que possuem uma produção inicial baixa influenciam tanto o seu crescimento como o dos municípios vizinhos. Além disso, como a dependência espacial foi detectada através dos resíduos, existem fatores não modelados que podem estar influenciando o processo de divergência da produtividade.

A partir dos resultados obtidos, foi possível constituir evidências de um modelo de divergência de produtividade agrícola dos municípios gaúchos. Ao comparar as estimativas dos parâmetros entre os subperíodos, observa-se que o valor do parâmetro nos anos de 2008-2015 chega a quase zero; no entanto, trata-se de um valor significativo e positivo. Dito isso, o estudo aponta para uma queda da divergência de produtividade agrícola dos municípios do Rio Grande do Sul.

Outro aspecto importante a ser destacado com relação à não identificação da hipótese de convergência absoluta para a produtividade agrícola no Rio Grande do Sul é que o estado apresenta estruturas produtivas diferenciadas, que resultam da especialização em determinada atividade na região ou no município, como é o caso da vitivinicultura nos municípios da Serra e da produção de soja em Palmeira das Missões e Cruz Alta (FEE, 2017).

Diferentemente dos demais trabalhos que estudam a convergência de renda per capita no Rio Grande do Sul, o presente estudo apontou para a divergência de produtividade. Almeida (2012) salienta que os estudos realizados a níveis municipais buscam captar a heterogeneidade, pois, quando tratados a níveis de microrregiões ou mesorregiões, não é possível captar tal característica, o que pode levar, inúmeras vezes, a resultados que não rejeitam a hipótese de convergência, quando na verdade ela deve ser rejeitada.

Portanto, diante das diversidades de taxas de crescimento da produtividade agrícola dos municípios do Rio Grande do Sul e suas peculiaridades econômicas e sociais, tornam-se relevantes políticas governamentais que possam contribuir para os problemas residuais dos municípios. 


\section{CONCLUSÕES}

O presente trabalho buscou identificar a ocorrência ou não da convergência da produtividade agrícola nos municípios do Rio Grande do Sul. Com o intuito de captar a dependência espacial, julgada existente pelos vários teóricos especialistas no tema, foi utilizada a técnica de análise espacial.

O diagnóstico exploratório de dados espaciais contribuiu para a identificação de autocorrelação espacial global positiva, ou seja, os municípios estão localizados próximo a outros com características produtivas comuns. Além disso, a análise LISA cluster possibilitou a visualização dos municípios com alta correlação positiva e negativa.

No decorrer dos períodos analisados, as principais mudanças identificadas foram relacionadas ao quadrante BB. No ano de 2008, havia menos municípios com tal característica, sendo que, no ano de 2015, as principais regiões que passaram a compor o padrão BB, situadas no Norte, Noroeste, Centro-Oeste e Sudoeste do Rio Grande do Sul, aumentaram significativamente sua produtividade. O cluster composto pelos municípios do quadrante AA ficou localizado nas regiões de Vacaria, Montenegro, Passo Fundo, Gramado-Canela, Porto Alegre e Caxias do Sul.

O principal fator que pode ter contribuído para o alcance de tais resultados diz respeito à proporção de lavouras permanentes e temporárias. As regiões enquadradas nos padrões AA (Caxias do Sul, Vacaria, Passo Fundo) são caracterizadas pela predominância de lavouras temporárias, enquanto as regiões identificadas no quadrante BB, principalmente no extremo Norte e Sul do estado, se destacam na produção de grãos (soja e arroz). Tais atividades estão sujeitas, e também são mais vulneráveis, aos efeitos ocasionados por intempéries climáticas, podendo comprometer os níveis de produtividade de uma safra inteira.

Ao contrário dos estudos já realizados para a convergência da renda do estado, o modelo econométrico espacial captou a tendência de divergência da produtividade agrícola dos municípios gaúchos, rejeitando a hipótese do trabalho referente à convergência da produtividade. No entanto, os parâmetros estimados sugerem uma queda da divergência entre os subperíodos de 2001 a 2015.

A não aceitação da hipótese de convergência absoluta para produtividade do setor agrícola demonstra que as divergências estão aumentando no decorrer do período analisado. Diversos são os fatores que podem estar contribuindo para o acirramento dessas desigualdades: a heterogeneidade dos municípios gaúchos, a especialização em diferentes sistemas produtivos, fatores institucionais e até mesmo fatores geográficos e climáticos, no caso da agropecuária.

Dessa forma, sugere-se a realização de estudos que analisem o processo de convergência condicional, para que seja possível identificar as possíveis causas dessa desigualdade na produtividade do estado. 


\section{REFERÊNCIAS}

ABRAMOVITZ, Moses. Catching up, forging ahead, and falling behind. The Journal of Economic History, v. 46, n. 02, p. 385-406, 1986. Disponível em: <https://econpapers.repec.org/article/cupjechis/v_3a46_3ay_3a1986_3ai _3a02_3ap_3a385-406_5f04.htm>. Acesso em: 20 maio 2018.

ALMEIDA, Eduardo Simões. Função de Produção Agropecuária Espacial. In: ANAIS DO XLIII CONGRESSO BRASILEIRO DE ECONOMIA, ADMINISTRAÇÃO E SOCIOLOGIA RURAL (SOBER). Ribeirão Preto, 2005. Disponível em: <https://sober.org.br/anais/>Acesso em: 24 abril 2018.

ALMEIDA, Eduardo Simões. Existe convergência espacial da produtividade agrícola no Brasil?. Revista de Economia e Sociologia Rural, V. 46, n. 1, p. 31-52, 2008. Disponível em: <http://www.scielo.br/scielo.php?script=sci_arttext\&pid=S010320032008000100002>. Acesso em: 15 abril 2018.

ALMEIDA, Eduado Simões Econometria Espacial Aplicada. 1 ed. Campinas: Editora Alínea, 2012.

ANSELIN, Luc. Exploring Spatial Data with GeoDa. A Work Book. Spatial Analysis Laboratory, University of Illinois. Center for Spatially Integrated Social Science. 2004-2005. Disponível em: <http://www.csiss.org/clearinghouse/GeoDa/geodaworkbook.pdf $>$. Acesso em: 20 maio 2018.

ATLAS DO DESENVOLVIMENTO SOCIOECONÔMICO DO RIO GRANDE DO SUL. Secretaria de Planejamento, Orçamento e Gestão. Disponível em: <http://www.atlassocioeconomico.rs.gov.br/>, 2017. Acesso em: 15 maio 2018.

BARRO, Robert; SALA-I-MARTIN, Xavier. "Convergence". Journal of Political Economy, V. 100, n.2, p. 223-251, 1992. Disponível em: <https://dash.harvard.edu/bitstream/handle/1/3451299/barro_converg ence.pdf?sequence $=4>$ Acesso em: 16 maio 2018 .

BARRO, Robert. J.; SALA-I-MARTIN, Xavier. Convergence across states and regions. Center Discussion Paper, No. 629, Yale University, Economic Growth Center, New Haven, CT, 1991. Disponível em: <http://www.econ.yale.edu/growth_pdf/cdp629.pdf $>$. Acesso em: 10 maio 2018.

BAUMOL WillianJ. Productivity growth, convergence, and welfare: what the long-run data how. American Economic Review, v. 76, n. 5, p. 1072-1085, 1986. Disponível em: <http://piketty.pse.ens.fr/files/Baumol1986.pdf>. Acesso em: 10 maio 2018. 
BAUMONT, Catherine; ERTUR, Cem.; LE GALLO, Julie. Geographic spillover and growth: a spatial econometric analysis for European regions. LATEC-Document de travail, Economie, v. 7, Jun./ 2000. Disponível em: <https://hal.archives-ouvertes.fr/hal-01526987/document> Acesso em: 12 maio 2018.

BAUMONT Catherine; ERTUR, Cem.; LE GALLO, Julie. Spatial Convergence Clubs and the European Regional Growth Process, 19801995.. In: Fingleton B. (ed.), European Regional Growth. Springer Verlag, Berlin, pp. 131-158, 2003. Disponível em: <https://www.researchgate.net/publication/289004687_Spatial_Converg ence_Clubs_and_the_European_Regional_Growth_Process1980-1995> .

Acesso em: 10 maio 2018.

BERTUSSI, Geovana L.; FIGUEIREDO, Lízia. D. Investigando a hipótese de convergência na América Latina e no leste asiático: uma abordagem de regressão quantílica. Belo Horizonte: UFMG/Cedeplar. Disponível: <https://econpapers.repec.org/paper/cdptexdis/td354.htm>. Acesso em: 20 maio 2018.

CHADDAD, Fábio. Economia e organização da Agricultura Brasileira. 1 ed. Elsevier Brasil, 2017.

DA SILVA, Ismael M. et al. Associação de dados espaciais: uma análise exploratória para desenvolvimento econômico do estado do Pará. Revista Teoria e Evidência Econômica. Ano 17, n. 36, p. 63-79, jan./jun. 2011. Disponível em: <https:/ / pdfs.semanticscholar.org/e23f/8cd9b5771c57580e562407651bde bf9d0625.pdf>. Acesso em: 10 maio 2018.

DALL' ERBA, Sandy. Productivity convergence and spatial dependence among Spanish regions. Texto para discussão REAL/UIUC. Illinois, 2003. Disponível em: <https://experts.illinois.edu/en/publications/productivity-convergenceand-spatial-dependence-among-spanish-reg >. Acesso em: 10 maio 2018.

FEEDADOS. Fundação de Economia e Estatística. Dados e Mapas. Disponível em: <http://feedados.fee.tche.br/feedados/>. Acesso em: 6 abril 2018.

FEE. Painel do Agronegócio no Rio Grande do Sul - 2017. Disponível em: <http://www.fee.rs.gov.br/wpcontent/uploads/2015/09/20150903painel-do-agronegocio-no-rs2017.pdf>. Acesso em: 20 abril 2018.

FERREIRA, Pedro C; ELLERY JÚNIOR, Roberto G. Convergência entre a renda per capita dos estados brasileiros. Revista de Econometria, Rio de Janeiro, v. 16, n. 1, p. 83-103, 1996. Disponível em: <https://bibliotecadigital.fgv.br/dspace/bitstream/handle/10438/439/0 00062981.pdf>. Acesso em: 10 maio 2018. 
FLORAX, Raymond. JGM; FOLMER, Hendrik; REY, Sergio J. Specification searches in spatial econometrics: the relevance of Hendry's methodology. Regional Science and Urban Economics, v. 33, n. 5, p. 557-579, 2003.

<https://asu.pure.elsevier.com/en/publications/specification-searchesin-spatial-econometrics-the-relevance-of-h>. Acesso em: 12 maio 2018.

FOCHEZATTO, Adelar; STÜLP, Valter. J. Análise da convergência da produtividade da mão-de-obra agropecuária entre os estados brasileiros: aplicação de matrizes de Markov, 1990-2000. Revista de Economia e Sociologia Rural. Piracicaba-SP, v. 46, n. 03, p. 739-765, jul./set. 2008. Acesso em: $\quad$ http://www.scielo.br/scielo.php?script=sci_arttext\&pid=S010320032008000300007>. Acesso em: 10 maio 2018.

GALOR, Oded., Convergece? Inferences from Theoretical Models. Economic Journal. Vol. 106, n437, pp. 1056-1069, 1996. Disponível em: <https://ideas.repec.org/a/ecj/econjl/v106y1996i437p1056-69.html>.

Acesso em: 15 maio 2018.

INSTITUTO BRASILEIRO DE GEOGRAFIA E ESTATÍSTICA (IBGE). Banco de dados do Sistema de Recuperação Automática (SIDRA). Disponível em: <http:/ / www.sidra.ibge.gov.br>, acessado em 07 fev. de 2018.

LOPES, Janete L. Avaliação do processo de convergência da produtividade da terra na agricultura brasileira no período de 1960 a 2001. Tese (Doutorado em Economia Aplicada) ESALQ/USP, 193 f. 2004. Disponível em: <https://www.teses.usp.br/teses/disponiveis/11/11132/tde-05052005162512/pt-br.php. >. Acesso em: 10 abril 2018.

MAGALHÃES, André; HEWINGS, Geoffrey. J. D. AZZONI, Carlos. R. Spatial dependence and regional convergence in Brazil. Discussion Papers, Federal Reserve Bank of Chicago, Federal Reserve Board of Governors or the University of Illinois, 2000. Disponível em: <http://citeseerx.ist.psu.edu/viewdoc/summary?doi=10.1.1.123.4279>.

Acesso em: 10 maio 2018.

MELO, Fernando B. H. Agricultura nos anos 80: perspectiva e conflitos entre objetivos de política. Estudos Econômicos, v. 10, n. 2, p. 57-101, maio/ago $1980 . \quad$ Disponível em: <https:// webcache.googleusercontent.com/search?q=cache:qrghJvTMZm 4J:https://seer.ufrgs.br/AnaliseEconomica/article/download/10658/629 $\underline{4+\& c d=1 \& h l=p t-B R \& c t=c \operatorname{lnk} \& g l=b r}>$. Acesso em: 08 abril 2018.

MONASTERIO, Leonardo. M; ÁVILA, Rodrigo. P. Uma análise espacial do crescimento econômico no Rio Grande do Sul, 1939 - 2001. Revista de Economia, Brasília- DF, V. 5, n.2, p. 269-296, 2004. Disponível em: <https://anpec.org.br/revista/vol5/vol5n2p269_296.pdf>. Acesso em: 13 maio 2018. 
OLIVEIRA, Cristiano A.; JACINTO, Paulo De A; GROLLI, Priscilla. A. Crescimento econômico e convergência com a utilização de regressões Quantílicas: Um estudo para os municípios do Rio Grande do Sul - 197001. Ensaios FEE, Porto Alegre, v. 28, Número Especial, p. 671-700, 2008.

PORSSE, Alexandre A. Dinâmica da desigualdade de renda municipal no Rio Grande do Sul: evidências da análise estatística espacial. Texto para discussão, FEE $n^{o}$ 42, out. 2008. Disponível em: <https://www.fee.rs.gov.br/tedes/dinamica-da-desigualdade-de-rendamunicipal-no-rio-grande-do-sul-evidencias-da-analise-estatisticaespacial/> Acesso em: 10 abril 2018

REY, Sergio. J.; MONTOURI, Brett D. US Regional Income convergence: a spatial econometric perspective. Regional Studies, v. 33, n. 2, pg. 143-156, 1999. Disponível em: <http://web.pdx.edu/ crkl/SEAUG/papers/Rey_RS99.pdf>. Acesso em: 12 jun. 2018.

ROMER, David. Macroeconomía Avançada. Ed. MC Graw Hill, 3ª ed., 2006.

SÁ BARRETO, Ricardo C; ALMEIDA, Eduardo. A contribuição do capital humano para crescimento econômico e convergência espacial do PIB per capita no Ceará. Economia do Ceará em Debate. Instituto de Pesquisa e Estratégia Econômica do Ceará (IPECE), p. 10-26, 2008. Disponível em: <http://www2.ipece.ce.gov.br/encontro/artigos_2008/1.pdf>. Acesso em: 15 maio 2018.

SALA-I-MARTIN, Xavier. The classical approach to convergence analysis. Economic Journal, vol. 106, issue 437, p. 1019-36, 1996. Disponível em: <https://econpapers.repec.org/article/ecjeconjl/v_3a106_3ay_3a1996_3ai _3a437_3ap_3a1019-36.htm> Acesso em: 09 abril 2018.

SOLOW, Robert M. A Contribution to the theory of economic growth. Quarterly Journal of Economics, v. 70, p. 65-94, 1956. Disponível em: $<$ http://piketty.pse.ens.fr/files/Solow1956.pdf $>$. Acesso em: 23 maio 2018.

QUAH, Danny. Empirics for economic growth and convergence. European Economic Review. v. 40, p. 1353-75, 1995. Disponível em : <https://www.sciencedirect.com/science/article/pii/0014292195000518> Acesso em: 15 abril 2018. 